\title{
LA GESTIÓN DEL AGUA EN ESPAÑA: EXPERIENCIAS PASADAS, RETOS FUTUROS*
}

\author{
ANTONIO FANLO LORAS \\ Catedrático de Derecho Administrativo \\ Universidad de La Rioja
}

SUMARIO

INTRODUCCIÓN.- PRIMERA PARTE: LOS CONDICIONAMIENTOS DEL MEDIO FÍSICO.SEGUNDA PARTE: EL MARCO LEGAL.- TERCERA PARTE: REFLEXIÓN FINAL.

Excma. Sra. Rectora Magnífica de la Universidad de La Rioja,

Excmo. Sr. Presidente del Gobierno de La Rioja,

Dignas Autoridades,

Señoras y Señores Doctores,

Señoras y señores,

\section{INTRODUCCIÓN}

Al serme encomendada esta lección de apertura del curso no me resultó difícil, en un año como el presente, decidir su contenido. Este había sido, hasta los terribles sucesos del 11 de septiembre, el año del Plan Hidrológico Nacional. La difusión de su Anteproyecto suscitó, como pocos asuntos, el interés del ciudadano medio y sonadas reacciones sociales. El pasado 6 de julio se publico la Ley del Plan, por la que se autoriza el trasvase de $1.050 \mathrm{hm}^{3} /$ año del río Ebro a diferentes cuencas mediterráneas. Una medida que generó y genera sentimientos contradictorios de rechazo y esperanza. Por eso mismo, hablar de la gestión del agua en este inicio de curso era para mí obligado.

\footnotetext{
* Lección inaugural de apertura del curso académico 2001-2002 de la Universidad de La Rioja.
} 
Pero había otras razones para elegir un tema como éste. El pasado 5 de marzo se cumplía el 75 aniversario de la creación de las Confederaciones Hidrográficas y de la constitución de la primera de ellas, la del Ebro. En 1926, en efecto, inició su andadura esta original fórmula organizativa, pionera en el Derecho comparado.

De otro lado, el pasado día 22 de diciembre, se publicó la conocida como Directiva marco de aguas que constituye un hito en la evolución del Derecho de aguas de la Unión Europea. Incorpora al acervo comunitario instituciones jurídicas arraigadas entre nosotros y otras novedosas de marcado carácter medioambiental. La gestión del agua adquiere tras la Directiva una extraordinaria complejidad y exigirá profundas reformas de los Derechos nacionales y, lo que es más importante, obligará a modificar arraigados hábitos y asumir una nueva cultura del agua.

Estos acontecimientos, como digo, y no sólo la aprobación del Plan Hidrológico Nacional, explican mi interés en reflexionar ante Vds. sobre el marco institucional de la gestión del agua en España, al que desde hace diez años he dedicado una parte importante de mi actividad investigadora.

La gestión del agua es compleja por la singularidad de este recurso natural, cuya abundancia no depende de la voluntad del hombre, sino que es aleatoria en el espacio y en el tiempo. Es compleja por el número de entidades de distinta naturaleza que participan en su gestión. Es compleja, en fin, porque requiere enfoques multidisciplinares de distintas ciencias y técnicas.

Al Derecho le cumple una tarea instrumental, de segundo grado. No depende de la Ley que exista agua. Pero la Ley ordena su utilización y conservación. La posesión y utilización del agua ha sido históricamente causa de conflictos sociales.

El texto ibérico escrito más extenso conservado en España -el conocido como «Bronce de Contrebia», hallado en Botorrita, localidad próxima a Zaragoza-, corresponde a una sentencia dictada el año 87 a. Cristo que pone fin a una disputa de aguas entre dos localidades. Dos milenios después, el Plan Hidrológico Nacional, parece que puede generar una intrincada conflictividad judicial como la habida secularmente.

Al Derecho, como técnica de ordenación y pacificación de la vida social, le incumbe proporcionar las instituciones que garanticen el uso pacífico y sostenible del agua, respetuoso con el medio ambiente.

Mi intención en esta lección no es otra que facilitarles las claves conceptuales del entramado institucional -júridico, por lo tanto- de la gestión del agua en España. Ayudarles a situar cada una de las piezas de este complejo, pero apasionante rompecabezas.

Con tal propósito, dividiré mi intervención en dos partes de desigual extensión: Los condicionamientos del medio físico (Primera Parte) y el marco legal de la gestión del agua (Segunda Parte); concluiré con una Reflexión final. 
PRIMERA PARTE

\section{LOS CONDICIONAMIENTOS DEL MEDIO FÍSICO}

Abordo, pues la cuestión relativa a los condicionamientos del medio físico.

El Derecho de aguas de un país está condicionado, sobre todo, por las características geofísicas de su territorio. Es una constatación que avalan los estudios de Derecho comparado. La abundancia o escasez de aguas del país conforman sus instituciones jurídicas.

Este principio es especialmente cierto en el caso de España, marcada por los desequilibrios temporales y territoriales en la distribución del agua. Severas sequías dejan paso a lluvias torrenciales que provocan graves inundaciones. Nuestros ríos padecen estiajes permanentes durante prolongados períodos. Desequilibrios que pueden potenciarse si realmente está produciéndose el anunciado cambio climático. La España húmeda y la España seca son el reflejo tópico de esa realidad que no deja de ser simplificadora, pues, existen sistemas de explotación deficitarios en toda la geografía española, también, en la cuenca del Ebro.

Debido a estas circunstancias, disponemos de un 8 por ciento de los recursos existentes de forma natural en la red hidrográfica, esto es, sin alterarla artificialmente, frente al 40 por ciento de los demás países europeos. Este dato encierra en sí mismo la clave de la gestión del agua en el último siglo: la política de obras hidráulicas, la política hidráulica defendida por Joaquín Costa y otros regeneracionistas. Política hecha operativa mediante ambiciosos proyectos nacionales con destino principal al regadío (traigo a su memoria los Planes de Obras Hidráulicas de 1902 y 1933), en cuya elaboración participaron muy activamente ilustrados ingenieros de caminos de los servicios hidrográficos del Ebro.

Gracias al extraordinario esfuerzo inversor realizado a lo largo de todo el siglo $\mathrm{XX}$ por diferentes gobiernos y regímenes políticos distintos -por la sociedad española, en definitiva- contamos con una red de 1.200 embalses de regulación y nuestra disponibilidad de agua alcanza de media un 40 por ciento de los recursos naturales, la que disponen los demás países europeos, sin hacer obras.

Por esta razón, el régimen jurídico de las aguas corre parejo con el de las obras hidráulicas. Porque, sin obras no hay agua o no la hay con la seguridad (técnica, económica y jurídica) que requieren los usos a los que va destinada. Esta realidad ha marcado la política hidráulica del siglo XX, que es ante todo, una política de obras, una política de oferta. Obras hidráulicas promovidas y financiadas en su mayor parte por el Estado que, abandonando su política abstencionista del siglo XIX, las asume como función pública propia.

Gracias a ellas contamos con $40.000 \mathrm{hm}^{3} /$ año de recursos regulados netos, cuyo consumo se distribuye de la siguiente manera: 15 por ciento, para agua de boca; 79 por ciento, para la agricultura; 6 por ciento, para la industria. Esta distribución del consumo -idéntica a los países que tienen nuestras mismas características físicaspermite identificar fácilmente los usos donde potencialmente es posible ahorrar agua mediante medidas que faciliten el uso eficiente y sostenible del agua. 
He hablado antes de desequilibrios territoriales en la disponibilidad del agua. Para corregirlos se ha aplicado una de las medidas de gestión más singulares y controvertidas: los trasvases entre cuencas. El del Tajo-Segura es el más importante de los existentes, que no el único, al que se suma ahora el del Ebro. Recuerdo que la política de trasvases a las cuencas mediterráneas fue prevista por primera vez en el Plan Nacional de Obras Hidráulicas de 1933, siendo Ministro D. Indalecio Prieto.

La política de obras hidráulicas no parece que pueda considerarse agotada. Todavía existen demandas no satisfechas, por carecer de las adecuadas infraestructuras. No ha de extrañar que el incremento de las disponibilidades de agua sea uno de los objetivos de los planes hidrológicos. Bien es verdad que el incremento de los recursos se puede alcanzar, también, mejorando la calidad del agua, mediante infraestructuras de saneamiento. La política de obras futura habrá de ser necesariamente selectiva y complementarla con una adecuada política de gestión de la demanda, promoviendo el ahorro y la eficiencia en el uso del agua.

Quiero insistir, no obstante, a contracorriente de ciertos planteamientos de moda, en la utilidad de las obras de regulación, que cumplen funciones múltiples. Además de satisfacer las demanda de abastecimiento, regadío, etc., garantizan la existencia de caudales ecológicos que corrigen la irregularidad estacional de nuestros ríos o permiten el control y laminación de avenidas [desde su implantación en la cuenca hidrográfica del Ebro se ha podido comprobar la bondad del Sistema Informático de Control de Avenidas, aplicado al vaciado/llenado programado de los diferentes embalses que ha evitado ya varias catástrofes].

Por esas razones no comparto en absoluto el juicio negativo sin matices de quienes consideran los embalses de regulación perjudiciales per se al medio ambiente. No pueden negarse sus impactos negativos. Pero esos efectos han de ser valorados globalmente en el conjunto del proyecto. Tales obras, donde se dan circunstancias de irregularidad temporal y territorial del régimen de lluvias, constituyen el principal, sino único, instrumento para asegurar la disponibilidad de agua en ausencia de otras alternativas viables. Su ejecución, no obstante, debe estar sujeta a rigurosos estudios de evaluación y se ha de compensar con generosidad a los afectados, de acuerdo con las recomendaciones del Informe de la Comisión Mundial de Presas.

A propósito de la Directiva marco, alguna consideración merece su machacona insistencia en los aspectos cualitativos del agua. En España, es imposible separarlos de los cuantitativos. Si no hay agua, no es posible hablar de su calidad. La gestión del agua no puede ser contemplada exclusivamente desde la perspectiva cualitativa, cuando falta o escasea el agua. Agua que depende fundamentalmente de las obras. Esta es una realidad no suficientemente contemplada por la Directiva marco, muy condicionada por las características hidrológicas de los países del Norte de Europa. Resulta, así, paradójico que la Directiva ignore la importancia cuantitativa que tiene el regadío en la agricultura de los países del Sur de Europa. Como sorprende que no saque consecuencia operativa alguna del objetivo de la Directiva de contribuir a «paliar los efectos de las inundaciones y sequías», objetivo que no figuraba en la propuesta originaria y que ha sido incluido a instancias de España.

Para concluir este apartado relativo al medio físico, no debemos olvidar la existencia de cuencas compartidas con Portugal, cuyo aprovechamiento conjunto ha 
sido objeto del reciente Convenio de Albufeira de 1998, ajustado anticipadamente a las exigencias de la Directiva Marco de aguas.

\section{SEGUNDA PARTE}

\section{EL MARCO LEGAL}

Paso a continuación a exponer la Segunda Parte relativa al marco legal de la gestión del agua.

Sería pretencioso por mi parte pretender ofrecerles en los escasos minutos de que dispongo un completo panorama del Derecho de aguas en España, rama que ha alcanzado un extraordinario desarrollo y madurez. En el texto escrito de la lección pueden encontrar Vds. una apretada síntesis así como indicaciones bibliográficas precisas para su mejor conocimiento, entre las que resulta imprescindible El Derecho de Aguas, del profesor Sebastián MARTIN RETORTILLO. Me limitaré a realizar un rápido recorrido por las principales instituciones que caracterizan el modelo español de gestión del agua.

A) Debo destacar, en primer lugar, la publificación del agua, que afecta a la trascendental cuestión de la propiedad del agua. La Ley de Aguas de 1985 declaró públicas todas las aguas, sean superficiales o subterráneas renovables. Como bienes integrantes del dominio público del Estado su asignación y uso requiere previa concesión administrativa. Los particulares sólo adquieren el derecho de aprovechamiento, no la propiedad del agua, salvo los derechos adquiridos al amparo de la legislación anterior que la ley respeta.

En consecuencia, la asignación del agua en España corresponde a la Administración, sin que intervenga el mercado. En «compensación» el agua, en sí misma, no tiene precio, es gratuita. Los precios que pagan los usuarios financian los servicios relacionados con el agua, pero no el agua. En esta gratuidad se halla el principal obstáculo dogmático para admitir el llamado «mercado de aguas», introducido en la reforma de 1999.

El extraordinario poder conferido a la Administración no se ha correspondido con una adecuada dotación de medios personales y materiales, lo que explica la falta de control de miles de pozos clandestinos y la sobreexplotación de acuíferos.

B) En segundo lugar, el agua además de bien productivo es, sobre todo, un recurso natural cuya calidad y la de los ecosistema vinculados a ella hay que proteger.

Es la dimensión medioambiental de la gestión del agua auspiciada por distintas Directivas europeas y exigencia del principio de utilización racional de todos los recursos naturales establecido en el art. 45 de la Constitución. La virtualidad de este principio ha quedado notablemente reforzada tras la reforma de 1999 y tiene múltiples manifestaciones. 
Tres son las técnicas principales de protección:

- El establecimiento de caudales ecológicos.

- La sujeción de los aprovechamientos a evaluación ambiental.

- La autorización de vertidos, que en modo alguno es una licencia para contaminar, sino que su objeto es garantizar el buen estado ecológico de las aguas.

La contaminación del agua es un gravísimo problema, agravado en España por la escasez de caudales circulantes en nuestros ríos. Las Directivas de sustancias peligrosas, de aguas residuales urbanas y la de nitratos, han establecido rigurosas obligaciones para depurar los vertidos urbanos e industriales, así como evitar la contaminación producida por tales sustancias.

Ese objetivo persigue el Plan Nacional y los Planes Regionales de Saneamiento y Depuración aprobados por las Comunidades Autónomas para cuya financiación han creado tributos ambientales. En el caso de La Rioja, la Ley de saneamiento y depuración de aguas residuales, aprobada el pasado año, ha reformado en profundidad la legislación anterior de 1994, perfeccionando el marco jurídico existente y estableciendo un modelo organizativo (el Consorcio de Aguas y Residuos) que incentiva la cooperación municipal y la gestión del ciclo integral del agua.

Reducir la contaminación debe ser tarea prioritaria. Es injustificable que los vertidos urbanos no se depuren. El sector industrial debe integrar el respeto del medio ambiente en los costes productivos, sin chantajes sociales injustificables. Deben aplicarse códigos de buenas prácticas agrarias para evitar la contaminación difusa por nitratos. La protección de la calidad del agua es función propia de las Administraciones y a ellas les corresponde liderar cambios profundos en las formas tradicionales de utilizar el agua.

C) En tercer lugar, la organización administrativa del agua, imprescindible para ejercer las importantes funciones reservadas a los poderes públicos (asignación de los recursos, protección de su calidad, promoción y explotación de obras hidráulicas). El Derecho de Aguas español ha hecho aportaciones fundamentales en este ámbito, asumidas internacionalmente. La gestión por cuencas; los organismos de cuenca como autoridad especializada, descentralizada y participada para la planificación y gestión del agua son principios que inspiran la configuración de las Confederaciones Hidrográficas.

Resulta imposible resumir en unas palabras la atractiva historia de las Confederaciones. Unicamente quiero recordar, en este momento y en esta tierra, que el primer esbozo de lo que fueron luego las Confederaciones fue hecho por D. Félix Martínez Lacuesta, a la sazón Presidente de la Diputación Provincial de Logroño, en la ponencia presentada al I Congreso Nacional de Riegos, celebrado en Zaragoza, en 1913, con el título «La conveniencia de constituir la Mancomunidad económica del Ebro».

La Confederaciones nacieron como una original fórmula para la construcción y explotación de obras hidráulicas y la gestión integrada y conjunta de los aprovechamientos de la cuenca con la participación de los interesados. Llegaron a considerarse una alternativa al modelo de organización burocrática del agua existente. Incluso, una alternativa al regionalismo político. 
Su historia posterior no puede ser más azarosa. Lo más significativo es que desde 1942 hasta 1979 -las fechas lo dicen todo- los usuarios no han tenido representación en ellas y que, desde 1958 hasta 1985, las funciones soberanas de administración del agua han correspondido a las Comisarías de Aguas, órgano de naturaleza burocrática.

Las actuales Confederaciones son fruto de esta complicada evolución. Su estructura orgánica, compleja, no parece adecuada totalmente a las nuevas necesidades de gestión del agua. Apunto algunos de sus más relevantes problemas:

-La fractura que existe entre la estructura burocrática, agrupada en torno a la Presidencia y el resto de órganos participativos.

- El impacto que tendrá en las Confederaciones la actividad de las sociedades estatales para la construcción y explotación de obras hidráulicas (caso de ACESA, en el Ebro), al sustraerles su función más característica.

- La insuficiente dotación de medios personales y materiales.

- El insatisfactorio encaje orgánico y funcional de las Comunidades Autónomas en la gestión del agua, que se manifiesta en la insuficiente articulación de sus competencias sectoriales relacionadas con el agua.

\section{D) En cuarto lugar, la función que cumplen los planes hidrológicos como instrumento de ordenación de la gestión del agua en la cuenca.}

La planificación por cuencas es otra de las aportaciones del Derecho español que asume la nueva Directivo marco de aguas. Los planes hidrológicos no son simples programas de obras sino instrumentos de naturaleza normativa que acomodan las previsiones genéricas de la ley a las condiciones singulares de cada cuenca. De ahí la importancia del plan, al que la Ley confía múltiples cometidos, entre otros, determinar el orden de prelación de los usos del agua.

Siendo piezas clave para la aplicación de la Ley, su elaboración ha sido lenta y su aprobación se ha demorado trece años. En el momento presente están aprobados todos los planes hidrológicos, salvo los de Galicia-Costa y Canarias. La árdua tarea realizada facilitará el cumplimiento futuro de los compromisos de la Directiva marco que son más ambiciosos que los establecidos por nuestra legislación (incluyen las aguas costeras y plazos perentorios para aprobar los planes y los programas de medidas, así como un análisis económico de los usos del agua).

La adopción del principio de planificación y gestión del agua por cuencas no es incompatible, con la existencia de un Plan Hidrológico Nacional. Su funcionalidad es, sin embargo, muy distinta de la de los planes de cuenca. El contenido principal de dicho Plan es la «previsión y condiciones» de los trasvases.

Como quiera que éstos rompen el principio de unidad de gestión por cuencas su aprobación está reservada al Parlamento y es una medida de carácter excepcional. En esos casos, la ley autoriza la toma de las aguas sobrantes de una cuenca y su envío a otra deficitaria. Concepto de «aguas sobrantes», «cuencas deficitarias», de determinación problemática y controvertida, como la experiencia del Tajo-Segura ha demostrado. 
Los trasvases son, antes que un asunto técnico o económico, una decisión esencialmente política sobre el modelo de desarrollo económico, social y territorial que se desea para el conjunto del país. Nuestra legislación de aguas, para facilitar la racionalización del proceso de decisión y encauzar jurídicamente los posibles conflictos, exige que junto a la autorización del trasvase se establezcan sus «condiciones». Se trata de un concepto jurídico cargado de exigencias, cuya inclusión en la Ley fue una aportación de parlamentarios aragoneses, meros transmisores de lo que significó en la opinión pública aragonesa el intento de trasvase a Barcelona en los años setenta y eco de razonables propuestas doctrinales. En tal sentido se había manifestado, en 1975, mi maestro el profesor Lorenzo Martín Retortillo, que ha tenido la amabilidad de acompañarnos en este acto.

Con independencia, pues, de la justificación técnica y política del trasvase del Ebro -extremo sobre el que no estoy capacitado para opinar- es importante para la cuenca cedente examinar las garantías establecidas en su condicionado. En este sentido, entiendo que la Ley del Plan establece un conjunto de garantías que parece suficiente en el plano formal. Destaco sólo tres:

$1^{\text {a }}$ Garantía de la demanda actual y futura de la cuenca cedente, sin limitación de su desarrollo derivado del trasvase.

$2^{a}$ Garantía en el lugar de toma del caudal mínimo ambiental fijado en el Plan Hidrológico de la cuenca del Ebro, más el correspondiente a las concesiones existentes aguas abajo de la toma. Provisionalmente, ese caudal mínimo es de $100 \mathrm{~m} 3 /$ segundo (art. 33.3 PH del Ebro).

$3^{\text {a }}$ Explotación de los embalses y sistemas hidráulicos por su órganos de gestión sin servidumbre alguna derivada del trasvase, salvo el caso de los de Mequinenza, Ribarroja y Flix.

Estas garantías podían haber sido, no obstante, más exigentes si se comparan con la Ley del Tajo-Segura, trasvase que técnicamente es muy distinto al del Ebro (uno en cabecera y otro en desembocadura). Por ejemplo, la Ley no fija por sí misma el volumen de agua trasvasada para cada uso sino que será el Gobierno quien lo determine. Tampoco establece un sistema de reservas mínimas en los embalses de cabecera, por considerar que éstos no tienen servidumbre alguna derivada del trasvase. En este sentido, los usuarios e interesados habrán de vigilar que la explotación de los embalses se realice, de verdad, sin hipotecas del trasvase.

Desde otro punto de vista, el régimen económico del trasvase parece poco generoso en compensaciones con la cuenca cedente.

El Plan Hidrológico Nacional ha sido aprobado por quien representa la soberanía del pueblo. Decisión absolutamente legítima mientras quien puede hacerlo no declare su disconformidad con la Constitución. A resultas de lo que en su día falle el Tribunal Constitucional, no debiera haber, en este conflicto, vencedores ni vencidos. La política del agua debe ser hoy escenario de una amplia concertación social y territorial donde se reconozca a cada parte, con generosidad, sus aportaciones. El desarrollo sostenible del arco mediterráneo debe ser compatible con la promoción de la España interior y, en particular, con las extraordinarias posibilidades del valle del Ebro, que tiene en el agua un recurso estratégico de primer orden. 
E) En quinto lugar, en relación con las obras hidráulicas me interesa destacar que las diversas reformas legislativas de los últimos años ponen de manifiesto un replanteamiento del papel del Estado en su construcción y financiación. La incorporación de un concepto más preciso de obras hidráulica de interés general, únicas que serán competencia del Estado (aunque desmentido por el largo Anexo II de la Ley del Plan Hidrológico Nacional), junto a las limitaciones presupuestarias y la utilización de fórmulas organizativas privadas (sociedades estatales) dan pie a esa conclusión. En este inicio del siglo XXI se abre un nuevo ciclo histórico de signo contrario a la estatalización de las obras hidráulicas asumida como función propia en los inicios del siglo XX, ante los fracasos reiterados de la iniciativa privada. Con las sociedades estatales de obras se abre una nueva etapa, un nuevo marco de relaciones jurídicas de base convencional, parcialmente privatizado y construido, por tanto, al margen de las previsiones generales de la legislación de aguas.

F) Finalmente una breves consideraciones acerca del régimen económicofinanciero del agua.

Se trata de una problemática con un alcance teórico y práctico de extraordinaria importancia. Ya he señalado que el agua como recurso no tiene precio, que es gratuita. $\mathrm{El}$ «precio» del agua remunera el coste de los servicios del agua (captación, conducción, abastecimiento y potabilización) o protegen su calidad (saneamiento). Así entendido, el régimen económico del agua tiene un alcance limitado, máxime si, como ocurre en nuestro Derecho, ese régimen está parcialmente subvencionado.

Me interesa destacar el auge de instrumentos económicos para fomentar el uso sostenible del agua. El principio de recuperación de costes los servicios de agua, incluidos los medioambientales y los del recurso recogido en la Directiva marco es una aplicación concreta de estas nuevas tendencias, de inmejorable intención, pero impregnadas, en mi opinión, de un rígido economicismo.

Su aplicación entre nosotros es motivo de preocupación, dado el importante peso que tienen entre nosotros las infraestructuras de regulación, imprescindibles para garantizar la oferta de agua. Se trata de un coste difícilmente comprensible para los países ricos en aguas. La Directiva admite excepciones al principio de recuperación del coste, cuyo alcance hay que justificar cumplidamente en los planes hidrológicos de la respectiva cuenca. En este sentido, entiendo que las obras de regulación cumplen finalidades ambientales y protectoras cuyo coste parcial debe asumirse por la colectividad. Incluso, el regadío debiera ser objeto de un tratamiento diferenciado. Una decisión de esa envergadura, sin embargo, no puede ser resuelta adecuadamente, sino es en el marco de la Política Agraria Comun.

\section{TERCERA PARTE}

\section{REFLEXIÓN FINAL}

Es el momento de concluir esta ya extensa lección. Lo haré con una breve reflexión final acerca del panorama que presenta la gestión del agua en España, de sus retos inmediatos. 
El panorama no puede ser más apasionante. En la superficie de la caudalosa corriente que es el Derecho de aguas se contempla un rico entramado institucional, que ha alcanzado su máximo desarrollo. Es la hora de la gestión, de hacer operativo el sistema institucional elaborado. Y la tarea es tan atractiva como inmensa.

El sentido y dirección de esta actividad no puede descubrirse sin comprender las corrientes profundas. Se habla de un cambio de paradigma en la gestión del agua, de una nueva cultura del agua. Del abandono del modelo de política de la oferta por el de gestión de la demanda.

Todo reduccionismo es simplificador. Es innegable, como ha señalado A. EMBID IRUJO, que las ideas-fuerza de la nueva cultura del agua se fundan en el valor primordial del medio ambiente, en la conservación de los recursos, en la gestión de la demanda, en la utilización de medios económicos para la gestión de un recurso escaso, en la formación en nuevas tecnologías, en la necesidad de incrementar el gasto en investigación y desarrollo en el ámbito del agua. Son valores asumidos por la Directiva marco de aguas.

Los responsables de la política del agua no debieran olvidar, sin embargo, los singulares condicionamientos físicos de nuestro país. Sería un error estratégico de imprevisibles consecuencias prescindir de aquellas técnicas que permiten el uso sostenible del agua, respetuoso con el medio ambiente.

Deseo profundamente que, como la lluvia fina, haya calado en Vds. el valor y la problemática del agua. El agua, bien precioso de la Naturaleza, al que el «poverello» de Asís, San Francisco, en el siglo XII, dedica un entrañable recordatorio en su Canto a las Criaturas:

«Loado seas, mi Señor, por la hermana agua,

La cual es muy útil y humilde, preciosa y casta

Y por nuestra hermana madre tierra, la cual nos sustenta y gobierna

y produce diversos frutos, con coloridas flores y hierbas»

Tierra, agua, frutos, hierbas, flores. Para eso sirve la gestión del agua. 